\title{
Food insecurity: What is the clinician's role?
}

\author{
Seth A. Berkowitz MD MPH, Gabriel E. Fabreau MD MPH
}

See also page E429 and www.cmaj.ca/lookup/doi/10.1503/cmaj.150234

$\mathrm{F}$ ood insecurity, or food poverty, describes uncertainty in consistently accessing nutritious food in socially acceptable ways. ${ }^{1}$ Although food insecurity is only one of several important social determinants of health, it is a common problem in many countries, including Canada (where $9 \%$ of households experience it $^{1}$ ), the United States and the United Kingdom. Food insecurity is associated with poor health, particularly cardiometabolic diseases such as obesity, diabetes and hypertension, as well as mental health disorders. ${ }^{2,3}$ There are few data, however, on its association with health care costs. In a linked research article, Tarasuk and colleagues ${ }^{4}$ help fill this important gap by examining annual health care costs by level of food insecurity.

Given widespread efforts to contain health care spending, and recognition that public policy programs may influence health indirectly, information about the costs associated with food insecurity is vitally important. Tarasuk and colleagues ${ }^{4}$ linked data on household food insecurity collected through the Canadian Community Health Survey to health expenditure data collected by the Ontario Ministry of Health and Long-Term Care. They found that annual health care costs in households with moderate or severe food insecurity were about \$500-\$1000 higher than costs in food-secure households. There was a "dose-response" pattern, with more severe food insecurity being associated with higher costs.

The cross-sectional design of the study precludes the evaluation of time ordering. The inability to determine whether food insecurity or health spending came first is an important limitation because of the strong potential for "reverse causation." Worsening health can make employment difficult and increase out-of-pocket healthrelated expenses. This can lead to food insecurity. Because Tarasuk and colleagues did not adjust for indicators of poor health or disease severity, we should be cautious about concluding that food insecurity causes increased health care spending. The association between food insecurity and poor health is likely complex, bi-directional and selfperpetuating: food insecurity may lead to poor health, worsening health can lead to worsening food insecurity, and the cycle continues. ${ }^{3}$

For clinical management, however, untangling causality might not be necessary. Adhering to a healthy diet is the foundation for preventing and treating cardiometabolic disease. However, food insecurity promotes consumption of highly processed, energy-dense foods laden with saturated fats, simple carbohydrates and sodium, because these foods are often cheaper than healthier alternatives. ${ }^{3}$ Whether or not food insecurity and poor health are causally related, once they coexist, food insecurity is clinically relevant.

A common question is whether routine screening for food insecurity with a validated instrument ${ }^{5}$ should be done in clinical settings. If the purpose of screening for food insecurity in the context of clinical care is to make health care more patient-centred, addressing food insecurity can be justified as simply the right thing to do. If the purpose is to improve health, however, the answer is more complicated. As in most cases of screening for health promotion, whether one should screen for food insecurity depends heavily on how the information will be used. No evidence yet shows that this type of screening improves health. Nevertheless, if routine screening for food insecurity changes clinical management, then it seems reasonable, especially given its low burden and cost.

Will screening for food insecurity change clinical management? There are situations where it clearly should. Patients with diabetes, for example, experience increased risk of hypoglycemia when food intake changes (as money

\section{- KEY POINTS}

- Food insecurity is common and linked to poor health outcomes.

- It is not known yet whether screening for food insecurity will improve health.

- Discussing food insecurity with patients is appropriate when it will change clinical management, and it may make care more patient-centred.

- In the absence of government-sponsored nutritional assistance programs, collaborations between community organizations and health care systems is a promising approach for addressing food insecurity. 
dwindles) through the month, but medication regimens do not. ${ }^{6} \mathrm{~A}$ clinician aware of this could offer explicit counselling and medication adjustment when food is limited, along with offering cost-conscious and culturally appropriate nutritional advice to all patients.

Beyond changing individual plans of care, screening for food insecurity could improve health if it were part of a program that connects patients to nutritional assistance. In Canada, there are no comprehensive government nutritional assistance programs. Instead, a patchwork of food banks and charitable agencies fills this gap. A recent survey of food banks found that program capacity has increased, but there is still substantial unmet demand. ${ }^{7}$ Health care systems could collaborate with community organizations to increase the support available for vulnerable patients. Community Food Centres Canada ${ }^{8}$ has resources to promote this collaboration and describes successful programs, including community gardens and kitchens, education and healthy-cooking programs, and Community Action Programs, such as the one in Toronto, that help empower marginalized food-insecure individuals. The project Improving Diabetes Care and Outcomes on the South Side of Chicago uses many of the same elements, including healthycooking classes, patient education, empowerment building and "food prescriptions" redeemable for discounts on produce. ${ }^{9}$ A report on the clinical results of this project is expected soon.

With demand increasing, food banks face substantial challenges in obtaining provisions, especially in the absence of government support. ${ }^{7}$ If health care systems work with food banks, clinicians could help ensure that the food provided promotes health, because many shelfstable foods are high in refined carbohydrates and sodium. In addition, clinicians could advocate for government-sponsored nutritional assistance or guaranteed income-supplement programs. A recent systematic review of the health effects of US food-subsidy programs found that participation in the programs was associated with dietary intake and health outcomes, especially birth weight. ${ }^{10}$

Another strategy is Food Is Medicine, ${ }^{11}$ whereby clinicians refer patients, as a covered health benefit, to organizations that provide medically tailored meals. This may sound unconventional, but it is already common to provide parenteral nutrition and enteral nutrition via tube feeding to promote health in both inpatient and outpatient settings. Providing prepared meals may also address other barriers to healthy eating that vulnerable patients face, such as intense demands on time placed by comorbidity, caregiving responsibilities and work, and knowledge of and equipment for food preparation. Future studies should determine whether medically tailored meals programs improve health, and if so, situations where they may be cost saving.

Food insecurity is a common problem, associated with both poor health and increased health care costs. Although we do not yet know whether these associations are causal, or whether routine screening for food insecurity will improve health, asking patients about food insecurity where it may change clinical management is appropriate. Studies to determine how best to overcome food insecurity will expand our ability to help patients who face this pernicious barrier to health.

\section{References}

1. Mikkonen J, Raphael D. Social determinants of health: the Canadian facts. Toronto: York University School of Health Policy and Management; 2010.

2. Laraia BA. Food insecurity and chronic disease. Adv Nutr 2013;4:203-12.

3. Seligman HK, Schillinger D. Hunger and socioeconomic disparities in chronic disease. N Engl J Med 2010;363:6-9.

4. Tarasuk V, Cheng J, de Oliveira C, et al. Association between household food insecurity and annual health care costs. CMAJ 2015;14:E429-36.

5. Hager ER, Quigg AM, Black MM, et al. Development and validity of a 2 -item screen to identify families at risk for food insecurity. Pediatrics 2010;126:e26-32.

6. Seligman HK, Bolger AF, Guzman D, et al. Exhaustion of food budgets at month's end and hospital admissions for hypoglycemia. Health Aff (Millwood) 2014;33:116-23.

7. Tarasuk V, Dachner N, Hamelin AM, et al. A survey of food bank operations in five Canadian cities. BMC Public Health 2014; $14: 1234$.

8. The Pod Knowledge Exchange [website]. Toronto: Community Food Centres Canada; 2015. Available: thepod.cfccanada.ca (accessed 2015 July 27).

9. Goddu AP, Roberson TS, Raffel KE, et al. Food Rx: a community-university partnership to prescribe healthy eating on the South Side of Chicago. J Prev Interv Community 2015;43:148-62.

10. Black AP, Brimblecombe J, Eyles H, et al. Food subsidy programs and the health and nutritional status of disadvantaged families in high income countries: a systematic review. BMC Public Health 2012;12:1099.

11. Ellwood M, Downer S, Leib EB, et al. Food Is Medicine: opportunities in public and private health care for supporting nutritional counseling and medically-tailored home-delivered meals. Cambridge (MA): Center for Health Law and Policy Innovation, Harvard Law School; 2014. Available: www.chlpi.org/wp-content/ uploads/2013/12/6.5.2014-Food-is-Medicine-Report-FINAL.pdf (accessed 2015 July 27).

Affiliations: Division of General Internal Medicine and Diabetes Population Health Research Center (Berkowitz), Massachusetts General Hospital; Harvard Medical School (Berkowitz), Boston, Mass.; Division of General Internal Medicine and Department of Community Health Sciences (Fabreau), University of Calgary, Calgary, Alta.

Contributors: Both authors contributed substantially to the conception and design of the manuscript. Seth Berkowitz drafted the article, and Gabriel Fabreau revised it critically for important intellectual content. Both authors approved the final version to be published and agreed to act as guarantors of the work. 